\title{
Mechanism of inhibiting type I interferon induction by hepatitis $B$ virus $X$ protein
}

\author{
Junyi Jiang ${ }^{1,2}$, Hong Tang ${ }^{1 凶}$ \\ ${ }^{1}$ Key laboratory of Infection and Immunity of Chinese Academy of Sciences, Institute of Biophysics, Beijing 100101, China \\ ${ }^{2}$ Graduate School of Chinese Academy of Sciences, Beijing 100049, China \\ $\bowtie$ Correspondence: tanghong@moon.ibp.ac.cn
}

Received November 21, 2010 Accepted December 2, 2010

\begin{abstract}
Hepatitis B virus (HBV) is regarded as a stealth virus, invading and replicating efficiently in human liver undetected by host innate antiviral immunity. Here, we show that type I interferon (IFN) induction but not its downstream signaling is blocked by HBV replication in HepG2.2.15 cells. This effect may be partially due to HBV $X$ protein (HBx), which impairs IFN $\beta$ promoter activation by both Sendai virus ( $\mathrm{SeV}$ ) and components implicated in signaling by viral sensors. As a deubiquitinating enzyme (DUB), HBx cleaves Lys63-linked polyubiquitin chains from many proteins except TANK-binding kinase 1 (TBK1). It binds and deconjugates retinoic acid-inducible gene I (RIG I) and TNF receptor-associated factor 3 (TRAF3), causing their dissociation from the downstream adaptor CARDIF or TBK1 kinase. In addition to RIG I and TRAF3, HBX also interacts with CARDIF, TRIF, NEMO, TBK1, inhibitor of kappa light polypeptide gene enhancer in B-cells, kinase epsilon (IKKi) and interferon regulatory factor 3 (IRF3). Our data indicate that multiple points of signaling pathways can be targeted by HBx to negatively regulate production of type I IFN.
\end{abstract}

KEYWORDS hepatitis B virus (HBV), HBV $X$ protein $(\mathrm{HBx})$, deubiquitination, type I interferon

\section{INTRODUCTION}

The innate immune system constitutes the first line of host defense against invading harmful microbes (Akira et al., 2006). In contrast to adaptive immune response, which relies on millions of antigen receptors generated by complex gene rearrangements, innate immune response is based on a limited number of germline-encoded pattern recognition receptors (PRRs) that sense pathogen associated molecular patterns (PAMPs) unique to microorganisms (Medzhitov, 2007). A variety of PRRs for virus-specific components encompassing viral nucleic acids are from three major classes: toll-like receptors (TLRs), retinoic acid-inducible gene I (RIG-I)-like receptors (RLRs), and nucleotide oligomerization domain (NOD)-like receptors (NLRs) (Kawagoe et al., 2009). NLRP3 (NALP3 or cryopyrin) and absent in melanoma 2 (AIM2) are known to regulate maturation of interleukin-1 (IL-1) family cytokines through activation of caspase 1 (Schroder et al., 2009), while engagement of other defined receptors and yet-to-be-identified IFN stimulatory DNA (ISD) sensors leads to induction of type I interferons (IFNs), proinflammatory cytokines and chemokines (Kawagoe et al., 2009; O'Neill, 2009; Sabbah et al., 2009). Type I IFNs, which consist mainly of a single $\beta$ and multiple $\alpha$ gene products, are crucial for antiviral defense and immune regulation (Honda et al., 2006). The binding of type I IFNs to the IFNAR (IFNa receptor) complex initiates Janus kinase/ signal transducer and activator of transcription (JAK/STAT) signaling cascade that results in expression of numerous IFNstimulated genes (ISGs), the products of which have a broad range of antiviral activities. In addition to conferring an antiviral state on cells, type I IFNs directly or indirectly activate dendritic cells (DCs), natural killer (NK) cells, T and B cells, providing a link between innate and adaptive immune response (McCartney and Colonna, 2009).

Hepatitis B virus (HBV), a member of the family Hepadnavirdae, is a hepatotropic, non-cytopathic, enveloped and partially double-stranded DNA virus that causes acute and chronic necroinflammatory liver diseases. Although highly effective prophylactic vaccines have been available since 1982 , it is estimated there are more than 350 million persistent carriers, $15 \%-40 \%$ of whom will develop cirrhosis, liver failure and hepatocellular carcinoma (HCC) (Lok and 
McMahon, 2007). HBV infection and its sequelae account annually for 1 million deaths worldwide (Dienstag, 2008).

Liver is the primary target of HBV, but very little is known about whether and how innate immune signaling pathways function in liver cells, especially in hepatocytes. The lack of detection of immune-related genes in the liver of infected chimpanzees has led to the consideration that HBV is a stealth virus (Wieland et al., 2004). However, this view seems to be contradicted by the observation that NK and NKT cells are promptly activated before the peak of virus expansion in natural human infection (Webster et al., 2000; Dunn et al., 2009; Fisicaro et al., 2009). Therefore, strategies may have been adapted by HBV to suppress the initial defenses of innate immunity, including type I IFN response. In line with the hypothesis, several HBV proteins, when overexpressed in cells, interfere with JAK-STAT signaling and ISGs expression: HBV surface proteins and/or $X$ protein $(\mathrm{HBX})$ upregulates protein phosphatase 2A (PP2A), which reduces the transcriptional activity of ISGF3 through inhibiting the enzyme activity of protein arginine methyltransferase 1 (PRMT1) (Christen et al., 2007); HBV polymerase inhibits nuclear translocation of STAT1 (Wu et al., 2007); HBV precore/core proteins prevent MXA gene expression via their interaction with the MXA promoter (Fernández et al., 2003). It should be noted that a virus may not be capable of spreading rapidly within a host's body because of the generation of antiviral state in neighboring cells, if the virus antagonizes IFN signaling but fails to limit IFN production (Randall and Goodbourn, 2008). Indeed, HBV core protein is indicated to repress the transcription of IFN $\beta$ (Whitten et al., 1991). Moreover, it has been shown that HBV virions, surface proteins and precore protein abrogate TLRselicited antiviral activity in mouse hepatocytes and nonparenchymal cells, which correlates with blocking the expression of IFN $\beta$ and ISGs (Wu et al., 2009).

Despite the progress that has been made, there is still much to be learned about the mechanisms through which HBV escapes type I IFN-mediated antiviral defense. Here we demonstrate that HBV replication inhibits type I IFN induction evoked by Sendai virus $(\mathrm{SeV})$ infection, whereas IFNARdependent signaling of IFN is not affected. HBx, a nonstructural protein encoded by HBV, might play an important role in suppression of host innate antiviral response by acting as a deubiquitinating enzyme. Furthermore, the specificity of $\mathrm{HBx}$ for signaling molecules attached with Lys63-linked polyubiquitin chains is broad, reflecting that $\mathrm{HBx}$ attenuates type I IFN response at multilevel, just as suggested by its interactions with various proteins.

\section{RESULTS}

\section{HBx inhibits type I IFN production}

Many viral nonstructural (NS) proteins, including hepatitis $C$ virus (HCV) NS3/4A and NS5A, respiratory syncytial virus
(RSV) NS1 and NS2, west nile virus (WNV) NS1, NS2A and NS4B and influenza A virus NS1, have been found to interfere with host innate immune response to aid virus replication and spread (Bowie and Unterholzner, 2008; Randall and Goodbourn, 2008). As the only unique NS protein of HBV, HBx is an enigmatic molecule because of its pleiotropic functions in regulating virus replication, cellular transcription, signal transduction, proteasome activity, cell cycle, apoptosis, tumor genesis and metastasis (Bouchard and Schneider, 2004). We hypothesized that HBx might also be involved in the inhibition of type I IFN production induced by SeV infection. To test this possibility, we infected HEK293T cells transiently expressing $\mathrm{HBx}$ with $\mathrm{SeV}$ and determined type I IFN response. Indeed, when HBx was overexpressed, SeVinduced activation of IFN $\beta$ promoter was dramatically inhibited (Fig. 1A). Following recognition of viral RNAs, TLR3 and RIG I/MDA5 associate with their respective adaptor molecule TRIF and CARDIF (also known as MAVS, IPS-1 or VISA) to initiate signaling pathways that converge at recruitment of TRAF3 protein, subsequently activating TANK-binding kinase 1 (TBK1)/inhibitor of kappa light polypeptide gene enhancer in B-cells, kinase epsilon (IKKi) kinases to phosphorylate interferon regulatory factor 3 (IRF3) and IRF7 (Kawagoe et al., 2009). Compared to RIGI and IRF3, which showed modest IFN $\beta$ promoter activation, overexpression of TRIF, CARDIF or TBK1 in HEK293T cells robustly induced the activation of IFN $\beta$ promoter (Fig. 1B-F). However, in each instance, co-expression of $\mathrm{HBx}$ suppressed IFN $\beta$ promoter activity in a dose dependent manner, probably indicating that $\mathrm{HBx}$ could function at least downstream of IRF3 (Fig. 1B-F). As a constitutively expressed transcription factor, IRF3 is essential for induction of type I IFN (Honda et al., 2006). Upon viral infection, it undergoes phosphorylation, dimerization and nuclear translocation sequentially to switch on gene expression. Consistent with the data above, IRF3 dimerization induced in SeV-infected HEK293T cells was impaired by overexpression of HBx (Fig. S1).

\section{HBx acts as a deubiquitinating enzyme to inhibit ubiquitination of IRF3 and IRF7}

Ubiquitination, one of the most important posttranslational modifications to which proteins in eukaryotic cells are subject, is extensively adopted to orchestrate appropriate immune responses against pathogens (Bhoj and Chen, 2009). Lys48linked polyubiquitin chains are generally associated with proteasomal degradation of target proteins, whereas Lys63linked chains participate in signal transduction and other processes (Hochstrasser, 2009). IRF3 was revealed to be modified with Lys63-linked polyubiquitin chains, which is important for its activation (Zheng et al., 2008; Zeng et al., 2009). To analyze the mechanism of HBx's function in innate immune response, we attempted to determine if $\mathrm{HBx}$ affected the ubiquitination of IRF3. After overexpressed green 

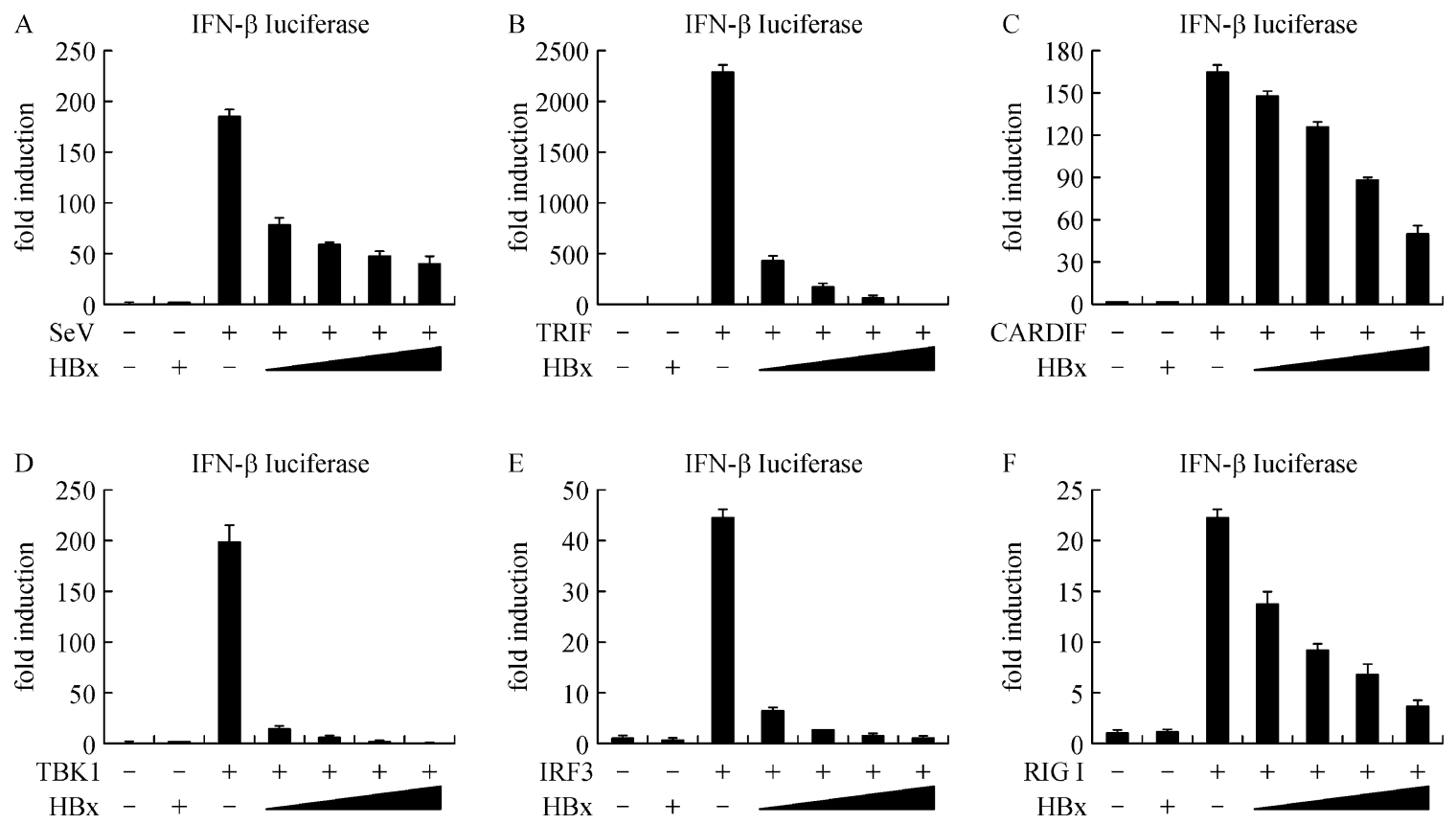

Figure 1. HBx interferes with type I interferon (IFN) induction. (A) Activation of an IFN $\beta$ luciferase reporter in HEK293T cells transfected with vectors or increasing amounts of $\mathrm{Myc}-\mathrm{HBx}$, and then infected for $12 \mathrm{~h}$ with Sendai virus (SeV). (B-F) Activation of an IFNß luciferase reporter in HEK293T cells transfected with FLAG-TRIF (B), Myc-CARDIF (C), HA-TBK1 (D), GFP-IRF3 (E) or FLAGRIG I (F), together with vectors or increasing amounts of Myc-HBx. Data are representative of three independent experiments (mean \pm s.d.). HA, hemagglutinin; GFP, green fluorescent protein; TBK1, TANK-binding kinase 1; IRF3, interferon regulatory factor 3; RIG I, retinoic acid-inducible gene $\mathrm{I}$.

fluorescent protein (GFP)-IRF3 was immunoprecipitated from cell lysates, smears corresponding to ubiquitinated IRF3 were detected with anti-GFP antibody (Fig. 2A). However, the ubiquitination of IRF3 was significantly reduced by $\mathrm{HBx}$ (Fig. 2A). IRF7 is another key regulator of type I IFN gene expression elicited by virus or TLR ligands (Honda et al., 2006), activation of which requires TRAF6-mediated ubiquitination (Kawai et al., 2004). As IRF7 is highly homologous to IRF3, we examined the effect of $\mathrm{HBx}$ on its ubiquitination. Similarly, HBx also reduced the amount of ubiquitinated IRF7 when hemagglutinin (HA)-tagged wild-type ubiquitin was expressed (Fig. 2B).

Ubiquitination can be reversed by deubiquitinating enzymes (DUBs), which are also implicated in modulating innate and adaptive immune system (Bhoj and Chen, 2009; Reyes-Turcu et al., 2009). DUB activities have been demonstrated in viral proteins such as OTU domain of $L$ (Nairoviruses) and NSP2 protein (Arteriviruses), PLpro/PLP2 domain of NSP3 protein (Coronaviruses) and UL36 USP homologs (Herpesviruses) (Randow and Lehner, 2009). To dissect further the inhibition of ubiquitination of IRF3 and IRF7, we assessed whether HBx can act as a DUB. Recombinant HBx or glutathione S-transferase (GST)-HBx purified from $E$. coli BL21 was incubated with Lys63-linked tetra-ubiquitin chains. HBx and GST-HBx both degraded Lys63-linked tetra-ubiquitin, although they were less effective than the positive control Isopeptidase T (IsoT) (Fig. 2C). The protease inhibitor $\mathrm{N}$-ethylmaleimide (NEM) inhibited the ability of HBx, GST-HBx and IsoT to cleave Lys63-linked tetra-ubiquitin chains, consistent with $\mathrm{HBx}$ being a cysteine protease (Fig. 2C).

\section{HBx deubiquitinates RIG I, RIG I-2CARD, TRAF3 and IKKi, but not TBK1}

Given that $\mathrm{HBx}$ is a DUB, it is probable that $\mathrm{HBx}$ can remove Lys63-linked polyubiquitin chains from other proteins besides IRF3 and IRF7 in the signaling cascades of type I IFN induction. The binding of Lys63-linked polyubiquitin chains synthesized by TRIM25 E3 ubiquitin ligase to RIG I, which results in and is reflected by RIG I polyubiquitination, is known to be crucial for cytosolic RIG I signaling in response to RNA virus infection (Gack et al., 2007; Zeng et al., 2010). As expected, the amount of ubiquitinated RIG I was decreased markedly in HEK293T cells co-transfected with Myc-HBx (Fig. 3A). The two $\mathrm{N}$-terminal caspase recruitment domains (CARDs) of RIG I are both responsible for binding of Lys63linked polyubiquitin chains (Zeng et al., 2010). When exogenous HA-ubiquitin was expressed, ubiquitination of RIG I-2CARD was more distinct than that of full length RIG I (Fig. 3B). In this experiment, we used anti-GST and anti-HA antibodies to probe ubiquitinated RIG I-2CARD. Considerable 
A

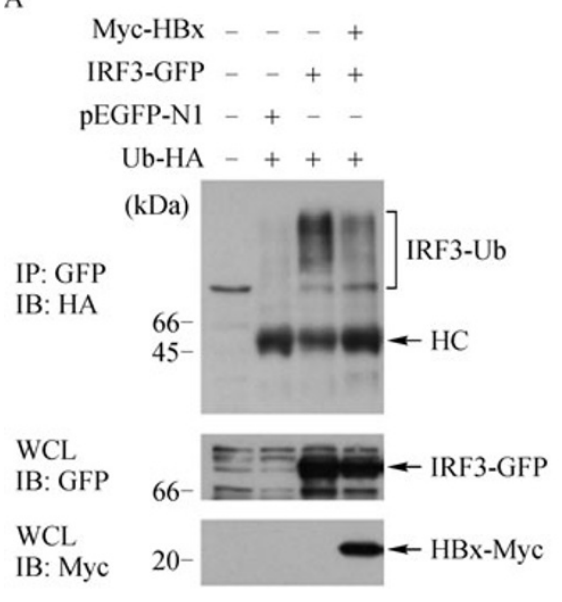

C

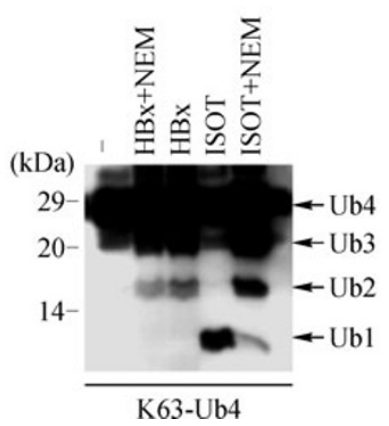

B

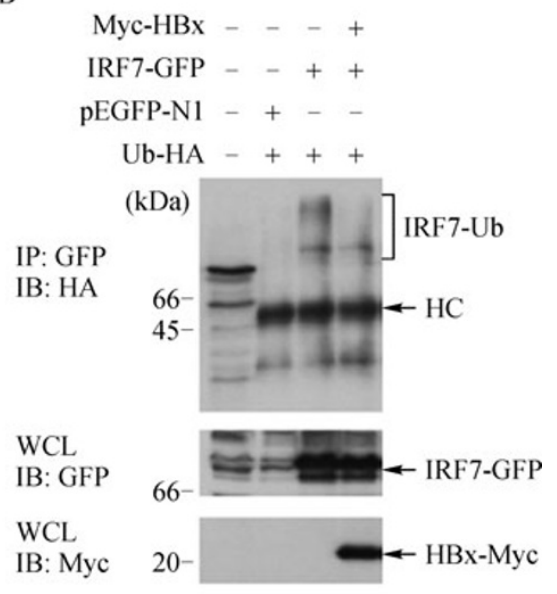

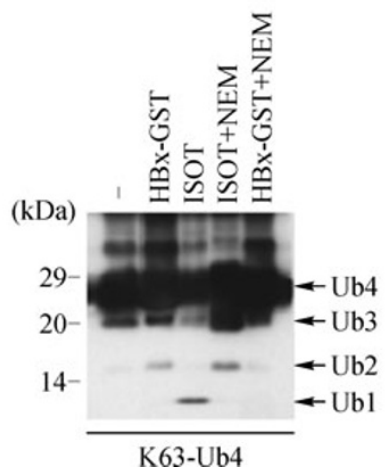

Figure 2. HBx is a deubiquitinating enzyme. (A and $B$ ) Immunoblot (IB) analysis of IRF3 (A) and IRF7 (B) ubiquitination (Ub), detected with anti-HA in anti-GFP immunoprecipitates of HEK293T cells transfected with GFP-IRF3 (A), GFP-IRF7 (B) or vectors (A and $B$ ), together with Ub-HA and Myc-HBx. WCLs (whole cell lysates) were examined by immunoblotting with anti-GFP and antiMyc, respectively. $\mathrm{HC}$, heavy chain. (C) Immunoblot analysis of ubiquitin isopeptidase activity of $\mathrm{HBx}$ in vitro. Recombinant protein HBx (left panel), GST-HBx (right panel) purified from E. coli or IsoT (positive control) was incubated with Lys63-linked tetra-ubiquitin (K63-Ub4) chains for $8 \mathrm{~h}$ at $37^{\circ} \mathrm{C}$. Products of deubiquitination reactions were analyzed by immunoblotting with anti-ubiquitin. Cysteine protease activity was blocked with $10 \mu \mathrm{M}$ (left panel) or $20 \mu \mathrm{M}$ (right panel) N-ethylmaleimide (NEM) in the reactions indicated. HBx, HBV X protein; GST, glutathione S-transferase; IsoT, Isopeptidase T. The other abbreviations are the same as in Fig. 1.

reduction of ubiquitination of RIG I-2CARD upon coexpression of $\mathrm{HBx}$ was observed in each case, corroborating HBx's DUB activity toward ubiquitinated RIG I (Fig. 3B). However, we also observed that the overall pattern of cellular protein ubiquitination was severely affected after $\mathrm{HBx}$ was cotransfected into cells (Fig. 3B). To further characterize the impairment of ubiquitin conjugates levels, we carried out deubiquitination assay with TRIM25 E3 ligase and endogenous ubiquitin. Whether anti-GST or anti-ubiquitin antibody was used, the significant decrease in ubiquitination confirmed again that RIG I-2CARD was a target substrate of $\mathrm{HBx}$ (Fig. S2). Similar to the data obtained with $\mathrm{HA}$-ubiquitin, $\mathrm{HBx}$ impaired the extent of ubiquitination in the cell, thus suggesting that the reduced modifications were attributed to the DUB activity of HBx (Fig. S2). Notably, enforced expression of RIG I-2CARD alone induced its low degree of conjugation with endogenous ubiquitin, which may contribute to its activation (Fig. 3B). Under such experiment conditions, TRIM25 E3 ligase strongly increased modification of RIG I2CARD, as evidenced by detection of the ubiquitinated forms even in the cell lysates (Fig. S2).

Next we investigated whether HBx could remove Lys63linked polyubiquitin chains from TRAF3, TBK1 and IKKi. Preventing the ubiquitination of TRAF3 (Kayagaki et al., 2007) and TBK1 (Wang et al., 2009), which are regulated by the deubiquitinase DUBA and E3 ubiquitin ligase Nrdp1, respectively, results in the sequestration of RLR-mediated signaling, whereas the function of ubiquitin modification on IKKi (Friedman et al., 2008) in production of type I IFN is still not clarified. Expression of HBx caused nearly complete loss of ubiquitination of TRAF3 and IKKi; by contrast, Lys63-linked polyubiquitin chains attached to TBK1 were not cleaved by 
A

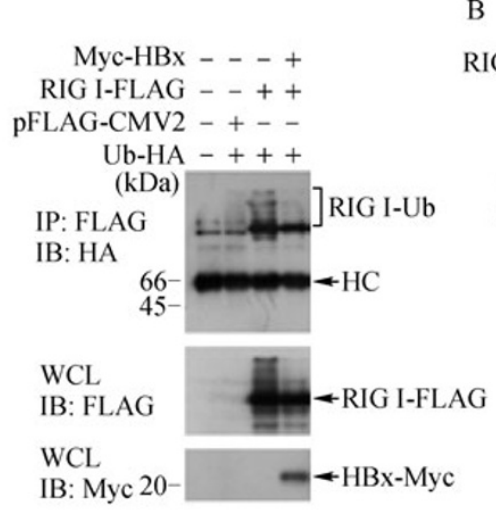

C

Myc-HBx - - +

TRAF3-YFP --++

pEGFP-N1 - + - -

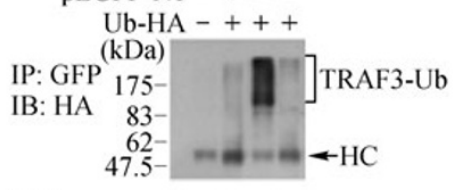

WCL

IB: GFP

WCL

IB: Myc

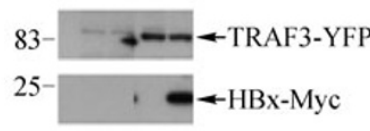

B

D

Myc-HBx - - - +

I-2CARD-GST --+++

pEBG -+--

Ub-HA -+-++

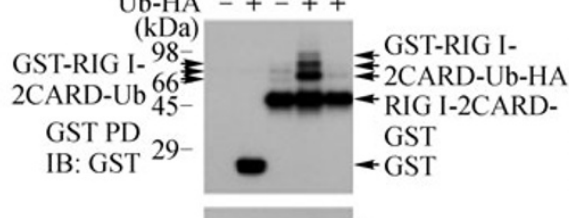

RIG I-2CARc-HBX $-\cdots+$

RIG I-2CARD-GST --+++ pEBG -+--

Ub-HA -+++

WCL $66^{-}-1$ RIG I-2CARD-
IB: GST $45^{-}-$GST
WCL $29-$ GST
IB: Myc 20-

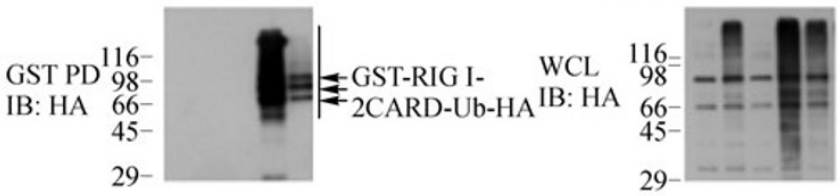

Myc-HBx - - - +

IKKi-FLAG --+++

pEF-FLAG B - + - - -

K63Ub-HA -+-++

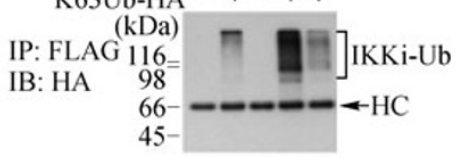

WCL

IB: FLAG

WCL

IB: Myc

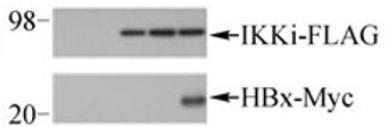

E

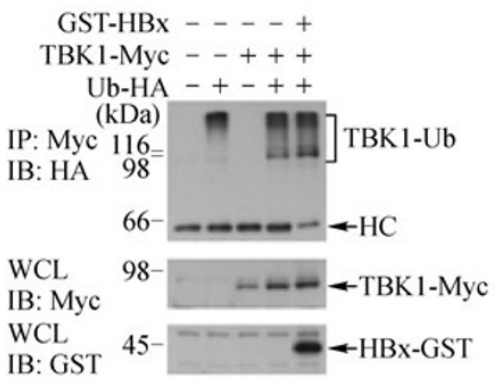

F

GST-HBx- - + - -

GST-HBx-N- - - + + -

GST-HBx-C- - - - +

FLAG-RIG I-2CARD -+++++

$\mathrm{Ub}-\mathrm{HA}+-++++$

$(\mathrm{kDa})$
IP: FLAG
$1168=$
98

WCL $45^{-} \cdot \quad$ a-

IB: GST

WCL
IB: FLAG

-HBX-N-GST

Figure 3. HBx displays DUB activity toward multiple proteins involved in type I IFN induction. (A-E) Immunoblot analysis of RIG I (A), RIG I-2CARD (B), TRAF3 (C), IKKi (D) and TBK1(E) ubiquitination. (A, C, and D) HEK293T cells were transfected with FLAG-RIG I (A), YFP-TRAF3 (C), FLAG-IKKi (D) or vectors, together with Myc-HBx and Ub-HA (A and C) or K63 Ub-HA (D), and incubated for $24 \mathrm{~h}$. RIG I, TRAF3 and IKKi were immunoprecipitated with anti-FLAG (A and D) or anti-GFP (C), and their ubiquitinations were assessed with an antibody specific for HA. (B) RIG I-2CARD were pulled down by GST beads from lysates of HEK293T cells transfected with pEBG or GST-RIG I-2CARD together with Myc-HBx and Ub-HA, and its ubiquitination was analyzed using anti-GST and anti-HA, respectively. (E) HEK293T cells were transfected with Myc-TBK1 and Ub-HA, together with GST-HBx. After $24 \mathrm{~h}$, lysates were prepared, and Myc-TBK1 was immunoprecipitated and probed with anti-HA. (F) Immunoblot analysis of antiFLAG immunoprecipitates from HEK293T cells co-transfected with FLAG-RIG I-2CARD, Ub-HA, and with either GST-HBx, GSTHBx-N or GST-HBX-C, detected with anti-HA. TRAF3, TNF receptor-associated factor 3; IKKi, inhibitor of kappa light polypeptide gene enhancer in B-cells, kinase epsilon; TBK1, TANK-binding kinase 1; HBx-N, N-terminal region of HBx; HBx-C, C-terminal region of $\mathrm{HBx}$. The other abbreviations are the same as in Fig. 1.

$\mathrm{HBx}$ (Fig. 3C-E). Taken together, our data indicate that HBx interferes with PRR signaling pathways at multiple points.

Based on the function in modulating gene transcription, $\mathrm{HBx}$ can be divided into two regions: $\mathrm{N}$-terminal negative regulatory domain (amino acids 1-50) and C-terminal transactivation or coactivation domain (amino acids 51-154)
(Tang et al., 2005). Subsequent analysis showed that the $\mathrm{N}$ terminal region $(\mathrm{HBx}-\mathrm{N})$ but not $\mathrm{C}$-terminal region $(\mathrm{HBx}-\mathrm{C})$ exhibited DUB activity toward ubiquitinated RIG I-2CARD (Fig. 3F). However, HBx-N disassemble polyubiquitin chains from RIG I-2CARD less effectively than did HBx (Fig. 3F). Therefore, full length, intact $\mathrm{HBx}$ is required for efficient 
function, albeit catalytic residues are mostly within N-terminal region.

\section{HBx associates with multiple proteins in signaling cascades of type I IFN induction}

To gain further insight into how HBx deubiquitinates RIG I and IRF3, we determined whether HBx interacts directly with RIG I and IRF3 to facilitate its function. Co-immunoprecipitation followed by immunoblot analysis demonstrated that both RIGI and IRF3 associated with $\mathrm{HBx}$, and $\mathrm{HBx}$ could be precipitated by $2 \mathrm{CARD}$ and $\triangle 2 \mathrm{CARD}$ regions of RIGI, respectively (Fig. 4A). Furthermore, in in vivo GST pull down experiments, the interactions between FIAG-tagged
RIG I or IRF3 and HBx were also detected, but only $\triangle 2 \mathrm{CARD}$ region of RIG I was indicated to bind $\mathrm{HBx}$ (Fig. S3A). To map the domain of $\mathrm{HBx}$ interacting with RIG I, we used the two $\mathrm{HBx}$ deletion constructions described above. It was found that $\mathrm{HBx}-\mathrm{C}$, but not HBx-N bearing DUB activity, is capable of binding full length RIG I (Fig. 4B and Fig. S3B). Similarly, the deconjugation of ubiquitin from TRAF3 and IKKi by $\mathrm{HBx}$ prompted us to analyze their physical associations with each other. We confirmed that $\mathrm{HBx}$ indeed interacted with TRAF3 and IKKi in HEK293T cells following either co-immunoprecipitation or GST pull down experiments (Fig. 4B and Fig. S3B). To our surprise, although $\mathrm{HBx}$ did not affect the ubiquitination of TBK1, an interaction between them was observed (Fig. 4B and Fig. S3B). In addition, we also noted that $\mathrm{HBx}$ was

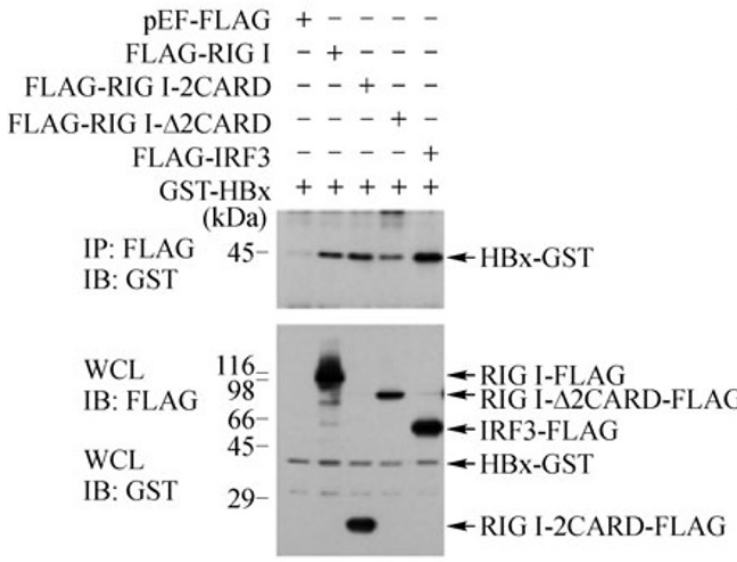

C

pEF-FLAG + - - -

FLAG-CARDIF - + - -

FLAG-TRIF - - + - -

FLAG-TANK - - +

FLAG-NEMO - - - +

GST-HBx +++++

IP: FLAG
IB: GST
WCL
pEF-FLAG + - + - -

FLAG-RIG I - + + - - -

GST-HBX-N - + - - -

GST-HBx-C + - + - -

GST-HBx - - + + + +

HA-FLAG-TBK1 $\ldots \ldots+\cdots$

FLAG-IKKi - - - - +

FLAG-TRAF3 $\ldots \ldots$

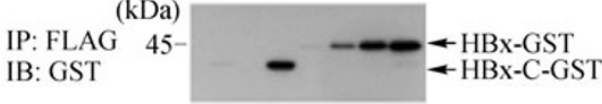

IB: GST - HBx-C-GST

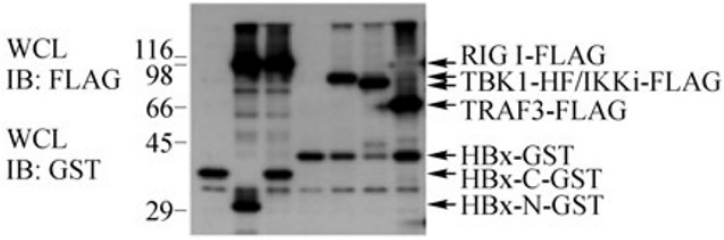

E
D

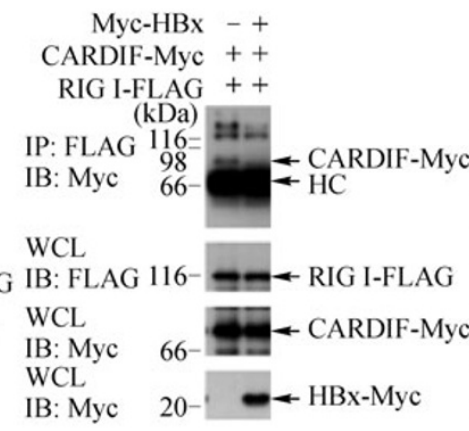

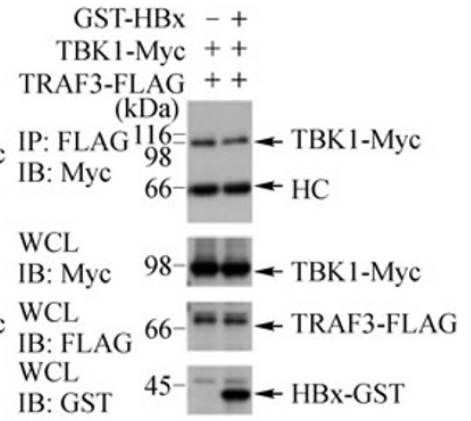

Figure 4. Interaction of HBx with diverse proteins crucial for type I IFN production. (A) Immunoblot analysis of proteins immunoprecipitated with anti-FLAG from lysates of HEK293T cells transfected with GST-HBx together with FLAG-tagged RIG I, RIG I-2CARD, RIG I- $\triangle 2 C A R D$, IRF3 or vectors, probed with anti-GST. (B) Immunoblot analysis of proteins immunoprecipitated with anti-FLAG from lysates of HEK293T cells transfected with GST-HBx, GST-HBx-N or GST-HBx-C together with FLAG-tagged RIG I, TBK1, IKKi, TRAF3 or vectors, probed with anti-GST. (C) Immunoblot analysis of proteins immunoprecipitated with anti-FLAG from lysates of HEK293T cells transfected with GST-HBx together with FLAG-tagged CARDIF, TRIF, TANK, NEMO or vector, probed with anti-GST. (D and E) Immunoblot analysis of proteins immunoprecipitated with anti-FLAG from lysates of HEK293T cells transfected with Myc- (D) or GST-HBx (E) together with either FLAG-RIG I and Myc-CARDIF (D) or FLAG-TRAF3 and Myc-TBK1 (E), probed with anti-Myc. The abbreviations are the same as in Fig. 1 and 2. 
associated with other proteins, such as CARDIF, TRIF and NEMO, which participate in activation of IRF transcription factors downstream of TLR3 and RIG I/MDA5 (Fig. 4C and Fig. S3C). In contrast, no binding of $\mathrm{HBx}$ was revealed to TANK, a scaffold protein functioning in vivo as a negative regulator of proinflammatory cytokine production instead of a regulator contributing to IFN responses (Kawagoe et al., 2009), likely suggesting it is favorable in the context of HBV infection (Fig. 4C and Fig. S3C). The binding and deubiquitination of RIG I and TRAF3 both implicate that HBx might interfere with their recruitments of downstream signaling components. In support of this speculation, overexpression of HBx reduced RIG I-CARDIF and TRAF3-TBK1 interaction, albeit to a lesser extent for the latter (Fig. 4D and 4E). In line with HBx's DUB activity toward diverse proteins, our data again indicate that signaling of type I IFN induction is manipulated by HBx at multilevel.

\section{HBV replication inhibits innate immune response to SeV}

In view of HBx's negative role in innate antiviral defense, it is probable that HBV replication could inhibit type I IFN production following virus infection. To investigate whether HBV replication influences innate immune response in hepatocytes, we exposed to SeV the hepatoma cell lines
HepG2, Huh7 and HepG2.2.15, a HepG2 derived cell clone stably transfected with replicative HBV genome. SeV was found to establish infections successfully in these cells as well as in the control $3 T 3$ cell line, which were confirmed by determination of the transcription of viral nucleocapsid (NC) gene using RT-PCR analysis (Fig. 5A and Fig. S4A). IFNAR engagement leads to tyrosine phosphorylation of STATs by JAK1 and tyrosine kinase 2 (TYK2) kinases in the type I IFN signaling pathway. In response to $\mathrm{SeV}$ infection, which activates endogenous cytosolic RIG I, HepG2 cells showed substantial phosphorylation of STAT1, while there was little if any phosphorylated STAT1 induced following SeV infection of HepG2.2.15 cells (Fig. 5B). In agreement to previous data suggesting that Huh7 cells respond weakly to SeV infection (Li et al., 2005), relatively low amounts of STAT1 were activated in Huh7 cells infected with $\mathrm{SeV}$ (Fig. 5B). To exclude the possibility that IFN mediated antiviral response was delayed in HepG2.2.15 cells, we analyzed the activation of STAT1 in the three cell lines at extended time points. Although phosphorylated STAT1 could still be detected in HepG2 cells at $24 \mathrm{~h}$ post infection, it remained undetectable in HepG2.2.15 cells (Fig. S4B). Collectively, these data indicate that replication of HBV inhibits innate immune response, including type I interferon, triggered by $\mathrm{SeV}$ infection.

To assess the effect of HBV replication on type I IFN
A

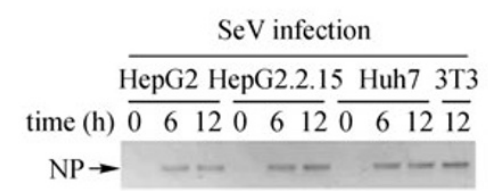
D
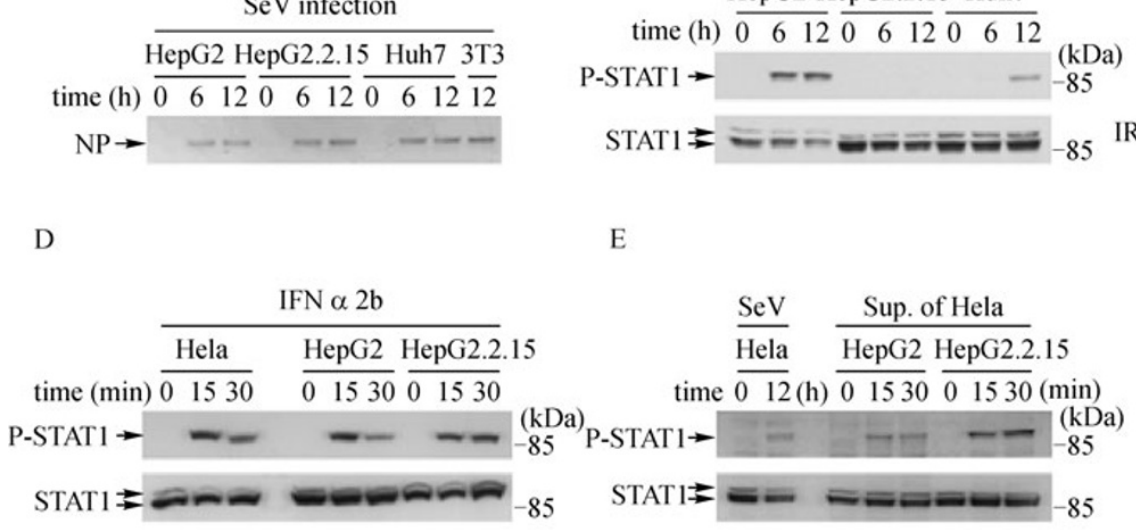

$\mathrm{C}$

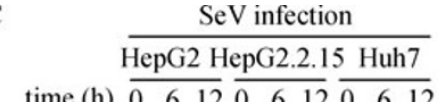

time (h) $\overline{0 \quad 612} \overline{0 \quad 6 \quad 12} \overline{0612}$

IRF3 dimer $\rightarrow$

F

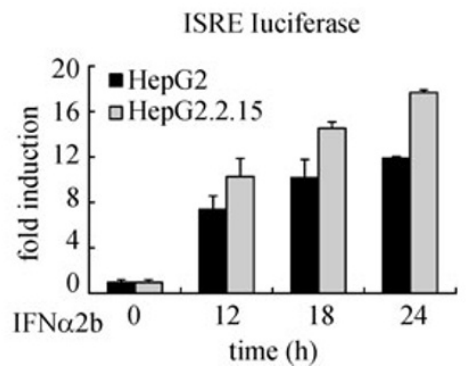

Figure 5. HBV replication inhibits type I IFN production in response to SeV. (A) RT-PCR analysis of NP expression in mock- or SeV-infected HepG2, HepG2.2.15, Huh7 and 3T3 cells (time, above lanes). (B) Immunoblot of lysates from hepatoma cells mockinfected or infected with SeV for various time (above lanes), detecting phosphorylation of STAT1 with antibody to phosphorylated ( $p$-) STAT1. (C) Native PAGE and immunoblot with anti-IRF3 of lysates from cells mock-infected or infected with SeV for various time (above lanes). (D and E) Immunoblot of lysates from HepG2 and HepG2.2.15 cells left untreated or stimulated with IFNa2b (D) or supernatants from Hela cells infected with SeV (E) for various time (above lanes) probed with anti-P-STAT1. Lysates of Hela cells left untreated or treated with IFNa2b (D) or SeV (E) were also analyzed as indicated. Total STAT1 (B, D, and E) serve as loading controls. (F) Activation of an ISRE luciferase reporter in HepG2 and HepG2.2.15 cells left untreated or stimulated with IFNa2b for various time is indicated at the bottom. Data are representative of three independent experiments (mean \pm s.d.). NP, SeV nucleoprotein; PAGE, polyacrylamide gel electrophoresis; IRF3, interferon regulatory factor 3; ISRE, IFN-stimulated response element. The abbreviations are the same as in Fig. 1 and 2. 
induction, we examined IRF3 activation following SeV infection. Unlike in HepG2 cells, we observed no dimerization of IRF3 induced by SeV in HepG2.2.15 cells, implicating that RIG I-mediated type I IFN production is suppressed by HBV replication (Fig. 5C). In addition, the absence of activated dimer form of IRF3 corroborates the weak response of Huh7 cells to SeV (Fig. 5C). To examine whether HepG2.2.15 cells are intrinsically defective in type I IFN signaling, we used IFNa2b as a stimulator to treat hepatoma cells. Activation of STAT1 occurred promptly in both HepG2 cells and HepG2.2.15 cells (Fig. 5D), and similar results were obtained after treatment of the two cell lines with culture supernatants from SeV-infected Hela cells (Fig. 5E). Further examination showed that IFN-stimulated response element (ISRE) promoter could also be activated by IFNa2b in HepG2 cells and HepG2.2.15 cells (Fig. 5F). Altogether, these data demonstrate that replication of HBV interferes with production of type I IFN rather than its downstream signaling cascades in hepatocytes.

\section{DISCUSSION}

Encoded by the smallest open reading frame of HBV, the regulatory protein $\mathrm{HBx}$ is able to exhibit pleiotropic biologic effects through modifying numerous signal transduction pathways (Bouchard and Schneider, 2004). However, whether $\mathrm{HBx}$ has a function in regulating innate antiviral response remains unclear. Here we have shown that $\mathrm{HBx}$ target multiple elements of the signaling cascades emanating from viral PRRs to suppress the induction of type I IFN. Specifically, we demonstrate that SeV-induced IFN $\beta$ promoter activation and IRF3 dimerization, as well as activation of IFN $\beta$ promoter by RIG I, CARDIF, TRIF, TBK1 and IRF3, is inhibited by $\mathrm{HBx}$. We establish that $\mathrm{HBx}$ is a DUB, which interacts and deubiquitinates proteins including RIG I, TRAF3, IKKi and IRF3. Apart from the four molecules, $\mathrm{HBx}$ also deconjugates IRF7 and associates with CARDIF, TRIF, NEMO and TBK1. Moreover, our data show that HBV replication suppressed SeV-induced type I IFN production in HepG2.2.15 cells, suggesting that $\mathrm{HBx}$ might be of importance in antagonizing host innate immunity, thus contributing to ensure efficient viral infection and propagation.

The mammalian immune system consists of innate and adaptive components, the cooperation of which protects the host from microbial infection and reinfection (Medzhitov, 2007). Despite the importance of innate immunity in controlling invading pathogens and relaying signals to the adaptive immune system, it seems to be silent in acutely HBV-infected chimpanzees (Wieland et al., 2004), which are partially attributable to specific replication strategy of HBV (Wieland and Chisari, 2005). Nonetheless, the lack of early intrahepatic antiviral genes induction may be peculiar to chimpanzee models, because NK and NKT cells are activated within $72 \mathrm{~h}$ after infection of woodchuck with WHV (woodchuck hepatitis virus), and results in transient suppression of viral replication (Guy et al., 2008). Moreover, IL-15 level and activation of NK and NKT cells are elevated during incubation phase of natural HBV infection, but tend to decline at the peak of viraemia, implying that HBV could counteract innate immune response (Dunn et al., 2009; Fisicaro et al., 2009). We have now provided evidence that production of type I IFN in response to $\mathrm{SeV}$ infection is dampened by HBV replication in HepG2.2.15 cells, when compared to that in HepG2 cells. In agreement with our data, a recent study has shown that IFN $\beta$ promoter activation induced by $\mathrm{SeV}$ was attenuated in HepG2.2.15 cells (Wang and Ryu, 2010). Replication of HBV does not have an effect on phosphorylation of STAT1 in cells stimulated with IFN (Christen et al., 2007), and we confirmed this result. However, we also found that signaling events downstream of STAT1 was not affected by HBV (Fig. 5F), which are contradictive to published studies that show nuclear translocation and DNA binding ability of STAT1 are impaired by HBV polymerase (Wu et al., 2007) and surface proteins and/or HBx (Christen et al., 2007) in Huh7 cells, respectively. Cell type specific differences might explain the apparent discrepancy. For example, HBx enhances HBV replication and secretion of surface and precore protein in HepG2 cells but not in Huh7 cells (Melegari et al., 1998). On the other hand, the decreased function of transcriptional coregulator PRMT1 on STAT1 methylation, which was reported to account for the reduction of transcriptional activity of STAT1 (Christen et al., 2007), is challenged by the finding that PRMT1 mediates arginine methylation of protein inhibitor of activated STAT1 (PIAS1) instead, leading to repression of IFN-inducible transcription via promoting the release of STAT1 from target gene promoter (Weber et al., 2009).

In parallel with phosphorylation, ubiquitination is of great importance in regulation of both the innate and the adaptive immune systems, so it is not surprising that viruses hijack the ubiquitin system to interfere with host immune response, including type I IFN induction. Consistent with this view, we demonstrated that $\mathrm{HBx}$, the multifunctional nonstructural protein of $\mathrm{HBV}$, was a DUB in nature, which could cleave Lys63-linked polyubiquitin chains from critical molecules, such as RIG I/RIG I-2CARD, TRAF3, IKKi, IRF3 and IRF7, involved in type I IFN signaling. The binding of Lys63-linked polyubiquitin chains to RIG I (Zeng et al., 2010) and Lys63linked polyubiquitination of TRAF3 (Kayagaki et al., 2007) are required for their individual binding to downstream complex containing CARDIF or TBK1, and subsequent antiviral signal transduction. HBx indeed attenuated RIG I-CARDIF and TRAF3-TBK1 interactions, but we were incapable of precluding the contributions of HBx's associations with these proteins (Fig. 4A-C and Fig. S3A-C). Although IKKi has been shown to undergo Lys63-linked polyubiquitination when coexpressed with ubiquitin (Friedman et al., 2008), whether this conjugation occurs under physiologic conditions is undefined, nor is its role in antiviral defense. However, it is 
possible that $\mathrm{HBx}$ deubiquitinates IKKi to inhibit IKKidependent activation of IRF3/IRF7, and/or phosphorylation of STAT1 on residue S708, regulating expression of a subset of ISGs required to contain viral load (Tenoever et al., 2007). Considering that activation of IRF3/IRF7 is an integration point of signaling of type I IFN induction (Honda et al., 2006; Kawagoe et al., 2009; O'Neill, 2009; Sabbah et al., 2009), the deubiquitination of IRF3/IRF7 by HBx seems to render HBV the capacity to intervene signal transduction by most sensors for viral nucleic acids. This speculation is further supported by the impairment of CARDIF, TRIF, TBK1 or IRF3-mediated IFN $\beta$ promoter activation in cells co-transfected with $\mathrm{HBX}$.

Conjugation of TBK1 with Lys63-linked polyubiquitin chains by Nrdp1, an E3 ubiquitin ligase interacted directly with TBK1, is essential for its optimal kinase activity toward IRF3 in TRIF and CARDIF-dependent signaling cascades (Wang et al., 2009). Unexpectedly, HBx did not reverse the Lys63-linked polyubiquitination of TBK1, but there was an interaction between the two proteins. It is well documented that DUBs can display specificity for both substrates and special types of ubiquitin chain (Reyes-Turcu et al., 2009). Although the action of $\mathrm{HBx}$ on Lys48-linked polyubiquitin chains was not determined, we found it disassembled polyubiquitin chains linked through K63 of ubiquitin and showed a broad substrate preference. Thus, the inability of $\mathrm{HBx}$ to deubiquitinate TBK1 might suggest that $\mathrm{HBx}$ requires additional partners to modulate its catalytic function in deconjugating some ubiquitinated proteins, which has been proved for many human DUBs.

The removal of Lys63-linked polyubiquitin chains from aforementioned molecules by $\mathrm{HBx}$ raises an issue of whether there are direct interactions between $\mathrm{HBx}$ and these proteins. By co-immunoprecipitation and in vivo GST pull down experiments, we obtained evidence that $\mathrm{HBx}$ was associated with RIG I, TRAF3, IKKi and IRF3. Furthermore, C-terminal transactivation domain of $\mathrm{HBx}$ and $\triangle 2 \mathrm{CARD}$ region of RIG I are crucial for their intermolecular associations. Whereas $\mathrm{N}$ terminal CARD-containing region of RIG I appeared to bind $\mathrm{HBx}$ in co-immunoprecipitation experiments, it could not be pulled down by GST-HBx, indicating that HBx might approach 2CARD domain through polyubiquitin chains or other adaptor protein. However, in comparison with RIG I-2CARD, the $\mathrm{K} 172 \mathrm{R}$ mutation, causing the former's marked loss of ubiquitination and poorly binding to CARDIF (Gack et al., 2007), did not reduce the amount of HBx precipitated (data not shown). A study has described that E3 ubiquitin ligase REUL-mediated attachment of Lys63-linked polyubiquitin chains to 2CARD domain at residues K154 and K164 is also necessary for eliciting RIG I signaling (Gao et al., 2009). Therefore, further experiments using RIG I-2CARD mutant, in which three ubiquitination sites are replaced, would help clarify whether ubiquitin chains influence HBx's binding to RIG I-2CARD. Moreover, it is perplexing that RIG I- $\triangle 2$ CARD interacts with $\mathrm{HBx}$. One possible explanation is that the direct interaction assists $\mathrm{HBx}$ in deconjugating $\triangle 2 \mathrm{CARD}$ region, the ubiquitination of which by E3 ubiquitin ligase RNF135 (Oshiumi et al., 2009) has been implicated in activation of RIG I. Notably, important components in TLRs and RLRsmediated signaling, such as CARDIF, TRIF and NEMO, the nuclear factor-kappaB (NF-kB) modulator acting upstream of TBK1/IKKi kinases in antiviral immunity (Zhao et al., 2007), also associate with $\mathrm{HBx}$. An exception is TANK, which serves as an essential positive adaptor bridging TRAF3 and TBK1/ IKKi in type I IFN response in vitro (Guo and Cheng, 2007) but not in vivo (Kawagoe et al., 2009). As an alternative, TANK negatively regulates canonical NF-kB signaling activated following injection of TLR ligands into Tank ${ }^{-1-}$ mice (Kawagoe et al., 2009). These results indicate that TANK seems to be advantageous to HBV for establishing infection, similarly to how SARM is thought to confer an advantage in vaccinia virus (VACV) infection (Bowie and Unterholzner, 2008).

As HBV replication in hydrodynamic mouse model and hepatocarcinogenesis in transgenic mice are promoted in the presence of $\mathrm{HBx}$, it is plausible that these processes may be characterized by inhibition of innate immune response by $\mathrm{HBx}$. In line with this notion, Pin1, which negatively regulates IRF3 signaling (Saitoh et al., 2006), is overexpressed prevalently in HBV-HCC positive for HBx (Pang et al., 2007). In addition, reduced CARDIF protein levels have been recently reported to correlate well with $\mathrm{HBx}$ expression in HBV-HCC (Wei et al., 2010). This downregulation is ascribed to the degradation of CARDIF promoted by HBx, providing another way by which HBx can interfere with RIG I/ MDA5 signaling (Wei et al., 2010). Nevertheless, it is somewhat controversial whether $\mathrm{HBx}$ influences production of type I IFN in the context of viral replication in HepG2 cells (Wang and Ryu, 2010; Wang et al., 2010; Wei et al., 2010). Further studies are required to understand the precise role and mechanisms of action of $\mathrm{HBx}$ in counteraction of innate antiviral response by HBV. In conclusion, our data show the intriguing function of $\mathrm{HBx}$ in limiting signaling pathways of type I IFN induction. By virtue of $\mathrm{HBx}$ and other molecules, such as polymerase (Wang and Ryu, 2010), HBV may evade and subvert innate immunity for its own benefit. Therefore, identification of HBx as an inhibitor of type I IFN might shed new light on HBV related liver diseases and represent potential therapeutic target in treatment of HBV infection.

\section{MATERIALS AND METHODS}

\section{Plasmid constructs}

Bacterial expression plasmid pGEX-6P-1-HBx and mammalian expression plasmids pEBG-HBx, pEBG-HBx-N (1-50 aa) and pEBG-HBx-C (51-154 aa) were generated by cloning PCR-amplified DNA fragments into pGEX-6P-1 or pEBG between restriction sites BamHI and Notl. For expression of Myc-tagged CARDIF, TBK1 and HA-FLAG-tagged TBK1, DNA fragments were cloned by PCR into 
Sall-Notl sites of pCMV-Myc or modified pCMV-HA. Using EcoRI and Sall, full length IRF3 sequence was subcloned into pCMV-FLAG2 to generate N-terminally FLAG-tagged expression construct. HBxC17SMyc mutant was obtained by PCR using site-directed mutagenesis. Other plasmids used were as follows: pCMV-Myc-HBx (H. S. Cho), pEF-Flag-RIG I-2CARD (1-229 aa), pEF-Flag-RIG-I- $\triangle 2 C A R D$ (218-925 aa) and pEF-Flag-RIG I (T. Fujita), pEBG-RIG I-2CARD (1-200 aa) and pEF-IRES-Puro-Trim25-V5 (J. U. Jung), pRK5-HAUbi and pRK5-HA-Ubi-K63 (K. L. Lim), pEGFP-IRF3 and pEGFPIRF7 (J. Hiscott), pEF-Flag-TRAF3 (N. Silverman), p3 × FLAGCMV10-CARDIF (C. M. Horvath), p3 x FLAG-CMV14-TRIF (M. K. Offermann), pcDNA3.1-FLAG-NEMO (A. Leonardi), pcDNA3.1FLAG-TANK, pcDNA3.1-FLAG-IKKi (A. Chariot), pEYFP-TRAF3, pEBB-HA-TBK1 and IFN $\beta$ luciferase reporter plasmid (G. $H$. Cheng), and ISRE luciferase reporter plasmid (H. B. Shu).

\section{Cell culture and transfection}

Human embryonic kidney (HEK293T), Hela, HepG2, Huh7 and mouse 3 T 3 cells were cultured in DMEM (Hyclone, Logan, UT, USA) supplemented with $10 \%(\mathrm{~V} / \mathrm{V})$ heat-inactivated fetal bovin serum (FBS) and 1\% penicillin-streptomycin. HepG2.2.15 cells were maintained in DMEM supplemented with $10 \%$ heat-inactivated FBS, $1 \%$ penicillin-streptomycin and $380 \mu \mathrm{g} / \mathrm{mL}$ G418. Cells were gown at $37^{\circ} \mathrm{C}$ in humidified air with $5 \% \mathrm{CO}_{2}$. Transient transfection of HEK293T cell was performed using the standard calcium phosphate method. HepG2 and HepG2.2.15 cells were transfected using Lipofectamine 2000 (Invitrogen, Carlsbad, CA, USA) transfection reagent according to the manufacturer's instructions.

\section{Reagents and antibodies}

Antibodies used were mouse monoclonal antibodies against $\beta$-actin (A5441, Sigma, Saint Louis, MO, USA), FLAG (F3165, Sigma), HA (SG4110-25, Shanghai Genomics, Inc, Shanghai, China), Myc (SG4110-18, Shanghai Genomics, Inc.), Ubiquitin (sc-8017, Santa Cruz, Santa Cruz, CA, USA) and GFP (33-2600, Zymed, San Francisco, CA, USA), rabbit polyclonal antibodies against GST (sc459, Santa Cruz), STAT1 (sc-346, Santa Cruz), IRF3 (sc-9082, Santa Cruz), phosphorylated STAT1 (9171, Cell Signaling, Danvers, MA, USA) and GFP (ab290, Abcam, Cambridge, MA, USA). Isopeptidase $\mathrm{T}$ (IsoT, E-322) and lysine63-linked tetra-ubiquitin (K63-Ub4, UC-310) chains were purchased from Boston Biochem (Cambridge, MA, USA). IFNa2b was kindly provided by Z. G. Su.

\section{Immunoprecipitation and immunoblot analysis}

Immunopreciptation and immunoblot analysis were performed as described previously (Zheng et al., 2008). IRF3 dimer was assessed by native polyacrylamide gel electrophoresis (PAGE), followed by immunoblot with anti-IRF3 antibody as described previously (Zhao et al., 2007).

\section{In vivo GST pull down}

In vivo GST pull downs were performed on cells transfected with vectors expressing GST, GST-RIG I-2CARD, GST-HBx, GST-HBx-N or GST-HBx-C. Post-centrifuged lysates were mixed with $50 \%$ slurry of Glutathione Sepharose 4B beads (Amersham Biosciences, Piscataway, NJ, USA) and incubated for $4 \mathrm{~h}$ at $4^{\circ} \mathrm{C}$. Beads were recovered and washed four times with lysis buffer before analysis by SDS-PAGE and immunoblot.

\section{Luciferase assay}

HEK293T, HepG2 or HepG2.2.15 cells seeded on 24-well plates were transiently transfected with $30 \mathrm{ng}$ of the IFN $\beta$ or ISRE luciferase reporter plasmid together with a total of $0.6 \mu \mathrm{g}$ various expression plasmids or empty control plasmids. As an internal control, $15 \mathrm{ng}$ of pRL-TK was transfected simultaneously. Then, $24 \mathrm{~h}$ later or at indicated time post-viral infection or IFNa2b stimulation, luciferase activity in the whole cell lysates was measured and normalized with a Dual-Luciferase Reporter Assay System (Promega, Madison, WI, USA).

\section{Viral infection}

Sendai virus (SeV) was from Wuhan Institute of Virology, Chinese Academy of Sciences (CAS) and was propagated in day 10 embryonated chicken eggs. The titer of virus stock prepared from allantoic cavity was determined by the pattern method for measuring hemagglutinin (HA) activity. Infection was performed in serum-free medium, and virus was added at a concentration of 25 hemagglutinating units per $\mathrm{mL}$. After $1 \mathrm{~h}$ incubation at $37^{\circ} \mathrm{C}$, the infecting medium was replaced with serum containing medium. Cells were harvested at appropriate time post infection and stored at $-80^{\circ} \mathrm{C}$ until analysis.

\section{RT-PCR and RNA extractions}

RNA extraction and reverse transcription were carried out as described previously (Zheng et al., 2008). One microliter of each cDNA template was incubated with Taq Polymerase (Roche) in subsequent PCR reactions of 25 cycles. The following primers were used for detection of $\mathrm{SeV}$ nucleoprotein (NP) gene: NP forward, 5'CGGAATTCGATG-TCGGGGATCGCCCTC-3'; NP reverse, 5'CGGGGTACCTTATGAGGGCGCAA-ACTTC-3'.

\section{Protein expression and purification}

$\mathrm{HBx}$ construct with an N-terminal GST tag was expressed in E. coli BL21 (DE3) cells (Novagen, Madison, WI, USA), utilizing a pET expression vector (Novagen). Cultures were grown at $37^{\circ} \mathrm{C}$ to an $\mathrm{OD}_{600}$ of 0.6 . Protein expression was induced by addition of $0.1 \mathrm{mM}$ IPTG, and cells were then incubated for $16 \mathrm{~h}$ at $4^{\circ} \mathrm{C}$ with shaking. Cells resuspended in $1 \times$ PBS were disrupted by sonication. After purification with Glutathione Sepharose $4 B$, recombinant protein was eluted by glutathione in elution buffer or cleaved by PreScission Protease in cleavage buffer according to the manufacturer's instructions.

\section{In vitro deubiquitination assay}

K63-Ub4 chains $(0.5 \mu \mathrm{g})$ were mixed with recombinant $\mathrm{HBx}(1 \mu \mathrm{g})$, GST-HBx $(1 \mu \mathrm{g})$ or IsoT in deubiquitination buffer $(50 \mathrm{mM}$ HEPES$\mathrm{NaOH}, \mathrm{pH} 8.0,10 \%$ glycerol, $5 \mathrm{mM}$ DTT), and incubated with/without 
10 or $20 \mu \mathrm{M} \mathrm{NEM}$ for $8 \mathrm{~h}$ at $37^{\circ} \mathrm{C}$. Reaction products were resolved by SDS-PAGE and then immunoblotted with anti-ubiquitin.

\section{ACKNOWLEDGEMENTS}

We thank H. S. Cho (Ajou University) for Myc-HBx; T. Fujita (Kyoto University) for Flag-RIGI, Flag-RIG I-2CARD and Flag-RIGI$\triangle 2 C A R D ;$ J. U. Jung (Harvard University) for GST-RIG I-2CARD and Trim25-V5; K. L. Lim (National Neuroscience Institute) for HA-Ub and HA-K63 Ub; J. Hiscott (McGill University) for GFP-IRF3 and GFPIRF7; N. Silverman (University of Massachusetts) for FLAG-TRAF3; C. M. Horvath (North-western University) for FLAG-CARDIF; M. K. Offermann (Emory University) for FLAG-TRIF; A. Leonardi (Federico II University of Naples) for FLAG-NEMO; A. Chariot (University of Liege) for FLAG-TANK, FLAG-IKKi; G. H. Cheng (University of California, Los Angeles) for YFP-TRAF3, HA-TBK1 and IFNß-luc; H. B. Shu (Wuhan University) for ISRE-luc; and Z. G. Su (Institute of Process Engineering, CAS) for reagent.

\section{ABBREVIATIONS}

CARDs, caspase recruitment domains; DCs, dendritic cells; DUBs, deubiquitinating enzymes; GFP, green fluorescent protein; GST, glutathione S-transferase; $\mathrm{HA}$, hemagglutinin; $\mathrm{HBV}$, hepatitis $B$ virus; $\mathrm{HBx}, \mathrm{HBV} X$ protein; HBx-C, C-terminal region of $\mathrm{HBx}$; $\mathrm{HBx}-\mathrm{N}, \mathrm{N}-$ terminal region of $\mathrm{HBx}$; $\mathrm{HCV}$, hepatitis $\mathrm{C}$ virus; IB, immunoblot; IFN, interferon; IKKi, inhibitor of kappa light polypeptide gene enhancer in B-cells, kinase epsilon; IRF3, interferon regulatory factor 3; ISD, IFN stimulatory DNA; JAK/STAT, Janus kinase/signal transducer and activator of transcription; ISGs, IFN-stimulated genes; IsoT, Isopeptidase T; ISRE, IFN-stimulated response element; NC, viral nucleocapsid; NF-kB, nuclear factor-kappaB; NK, natural killer; NLRs, nucleotide oligomerization domain (NOD)-like receptors; NP, SeV nucleoprotein; NS, nonstructural; PAGE, polyacrylamide gel electrophoresis; PAMPs, pathogen associated molecular patterns; PIAS1, protein inhibitor of activated STAT1; PP2A, protein phosphatase 2A; PRRs, pattern recognition receptors; PRMT1, protein arginine methyltransferase 1; RIG I, retinoic acid-inducible gene I; RLRs, RIGI-like receptors; SeV, Sendai virus; RSV, respiratory syncytial virus; TBK1, TANK-binding kinase 1; TLRs, toll-like receptors; TRAF3, TNF receptor-associated factor 3; TYK2, tyrosine kinase 2; $\mathrm{Ub}$, ubiquitination; WNV, west nile virus

\section{REFERENCES}

Akira, S., Uematsu, S., and Takeuchi, O. (2006). Pathogen recognition and innate immunity. Cell 124, 783-801.

Bhoj, V.G., and Chen, Z.J. (2009). Ubiquitylation in innate and adaptive immunity. Nature 458, 430-437.

Bouchard, M.J., and Schneider, R.J. (2004). The enigmatic X gene of hepatitis B virus. J Virol 78, 12725-12734.

Bowie, A.G., and Unterholzner, L. (2008). Viral evasion and subversion of pattern-recognition receptor signalling. Nat Rev Immunol 8, 911-922.

Christen, V., Duong, F., Bernsmeier, C., Sun, D., Nassal, M., and Heim, M.H. (2007). Inhibition of alpha interferon signaling by hepatitis B virus. J Virol 81, 159-165.

Dienstag, J.L. (2008). Hepatitis B virus infection. N Engl J Med 359, $1486-1500$
Dunn, C., Peppa, D., Khanna, P., Nebbia, G., Jones, M., Brendish, N., Lascar, R.M., Brown, D., Gilson, R.J., Tedder, R.J., et al. (2009). Temporal analysis of early immune responses in patients with acute hepatitis B virus infection. Gastroenterology 137 , 1289-1300

Fernández, M., Quiroga, J.A., and Carreño, V. (2003). Hepatitis B virus downregulates the human interferon-inducible MxA promoter through direct interaction of precore/core proteins. J Gen Virol 84, 2073-2082.

Fisicaro, P., Valdatta, C., Boni, C., Massari, M., Mori, C., Zerbini, A., Orlandini, A., Sacchelli, L., Missale, G., and Ferrari, C. (2009). Early kinetics of innate and adaptive immune responses during hepatitis B virus infection. Gut 58, 974-982.

Friedman, C.S., O'Donnell, M.A., Legarda-Addison, D., Ng, A., Cárdenas, W.B., Yount, J.S., Moran, T.M., Basler, C.F., Komuro, A., Horvath, C.M., et al. (2008). The tumour suppressor CYLD is a negative regulator of RIG-I-mediated antiviral response. EMBO Rep 9, 930-936.

Gack, M.U., Shin, Y.C., Joo, C.H., Urano, T., Liang, C., Sun, L., Takeuchi, O., Akira, S., Chen, Z., Inoue, S., et al. (2007). TRIM25 RING-finger E3 ubiquitin ligase is essential for RIG-I-mediated antiviral activity. Nature 446, 916-920.

Gao, D., Yang, Y.K., Wang, R.P., Zhou, X., Diao, F.C., Li, M.D., Zhai, Z.H., Jiang, Z.F., and Chen, D.Y. (2009). REUL is a novel E3 ubiquitin ligase and stimulator of retinoic-acid-inducible gene-l. PLoS One 4, e5760.

Guo, B., and Cheng, G. (2007). Modulation of the interferon antiviral response by the TBK1/IKKi adaptor protein TANK. J Biol Chem 282, 11817-11826.

Guy, C.S., Mulrooney-Cousins, P.M., Churchill, N.D., and Michalak, T. I. (2008). Intrahepatic expression of genes affiliated with innate and adaptive immune responses immediately after invasion and during acute infection with woodchuck hepadnavirus. J Virol 82, 8579-8591.

Hochstrasser, M. (2009). Origin and function of ubiquitin-like proteins. Nature 458, 422-429.

Honda, K., Takaoka, A., and Taniguchi, T. (2006). Type I interferon [corrected] gene induction by the interferon regulatory factor family of transcription factors. Immunity 25, 349-360.

Kawagoe, T., Takeuchi, O., Takabatake, Y., Kato, H., Isaka, Y., Tsujimura, T., and Akira, S. (2009). TANK is a negative regulator of Toll-like receptor signaling and is critical for the prevention of autoimmune nephritis. Nat Immunol 10, 965-972.

Kawai, T., Sato, S., Ishii, K.J., Coban, C., Hemmi, H., Yamamoto, M., Terai, K., Matsuda, M., Inoue, J., Uematsu, S., et al. (2004). Interferon-alpha induction through Toll-like receptors involves a direct interaction of IRF7 with MyD88 and TRAF6. Nat Immunol 5, 1061-1068.

Kayagaki, N., Phung, Q., Chan, S., Chaudhari, R., Quan, C., O'Rourke, K.M., Eby, M., Pietras, E., Cheng, G., Bazan, J.F., et al. (2007). DUBA: a deubiquitinase that regulates type I interferon production. Science 318, 1628-1632.

Li, K., Chen, Z., Kato, N., Gale, M. Jr, and Lemon, S.M. (2005). Distinct poly $(\mathrm{I}-\mathrm{C})$ and virus-activated signaling pathways leading to interferon-beta production in hepatocytes. J Biol Chem 280, 16739-16747.

Lok, A.S., and McMahon, B.J. (2007). Chronic hepatitis B. Hepatology 45, 507-539. 
McCartney, S.A., and Colonna, M. (2009). Viral sensors: diversity in pathogen recognition. Immunol Rev 227, 87-94.

Medzhitov, R. (2007). Recognition of microorganisms and activation of the immune response. Nature 449, 819-826.

Melegari, M., Scaglioni, P.P., and Wands, J.R. (1998). Cloning and characterization of a novel hepatitis $B$ virus $x$ binding protein that inhibits viral replication. J Virol 72, 1737-1743.

O'Neill, L.A. (2009). DNA makes RNA makes innate immunity. Cell 138, 428-430.

Oshiumi, H., Matsumoto, M., Hatakeyama, S., and Seya, T. (2009). Riplet/RNF135, a RING finger protein, ubiquitinates RIG-I to promote interferon-beta induction during the early phase of viral infection. J Biol Chem 284, 807-817.

Pang, R., Lee, T.K., Poon, R.T., Fan, S.T., Wong, K.B., Kwong, Y.L., and Tse, E. (2007). Pin1 interacts with a specific serine-proline motif of hepatitis B virus X-protein to enhance hepatocarcinogenesis. Gastroenterology 132, 1088-1103.

Randall, R.E., and Goodbourn, S. (2008). Interferons and viruses: an interplay between induction, signalling, antiviral responses and virus countermeasures. J Gen Virol 89, 1-47.

Randow, F., and Lehner, P.J. (2009). Viral avoidance and exploitation of the ubiquitin system. Nat Cell Biol 11, 527-534.

Reyes-Turcu, F.E., Ventii, K.H., and Wilkinson, K.D. (2009). Regulation and cellular roles of ubiquitin-specific deubiquitinating enzymes. Annu Rev Biochem 78, 363-397.

Sabbah, A., Chang, T.H., Harnack, R., Frohlich, V., Tominaga, K., Dube, P.H., Xiang, Y., and Bose, S. (2009). Activation of innate immune antiviral responses by Nod2. Nat Immunol 10, 1073-1080.

Saitoh, T., Tun-Kyi, A., Ryo, A., Yamamoto, M., Finn, G., Fujita, T., Akira, S., Yamamoto, N., Lu, K.P., and Yamaoka, S. (2006). Negative regulation of interferon-regulatory factor 3-dependent innate antiviral response by the prolyl isomerase Pin1. Nat Immunol 7, 598-605.

Schroder, K., Muruve, D.A., and Tschopp, J. (2009). Innate immunity: cytoplasmic DNA sensing by the AIM2 inflammasome. Curr Biol 19, R262-R265.

Tang, H., Delgermaa, L., Huang, F., Oishi, N., Liu, L., He, F., Zhao, L., and Murakami, S. (2005). The transcriptional transactivation function of $\mathrm{HBx}$ protein is important for its augmentation role in hepatitis B virus replication. J Virol 79, 5548-5556.

Tenoever, B.R., Ng, S.L., Chua, M.A., McWhirter, S.M., GarcíaSastre, A., and Maniatis, T. (2007). Multiple functions of the IKKrelated kinase IKKepsilon in interferon-mediated antiviral immunity. Science 315, 1274-1278.

Wang, C., Chen, T., Zhang, J., Yang, M., Li, N., Xu, X., and Cao, X. (2009). The E3 ubiquitin ligase Nrdp1 'preferentially' promotes TLR-mediated production of type I interferon. Nat Immunol 10, 744-752.

Wang, H., and Ryu, W.S. (2010). Hepatitis B virus polymerase blocks pattern recognition receptor signaling via interaction with DDX3: implications for immune evasion. PLoS Pathog 6, e1000986.

Wang, X., Li, Y., Mao, A., Li, C., Li, Y., and Tien, P. (2010). Hepatitis B virus $X$ protein suppresses virus-triggered IRF3 activation and IFNbeta induction by disrupting the VISA-associated complex. Cell Mol Immunol 7, 341-348.

Weber, S., Maass, F., Schuemann, M., Krause, E., Suske, G., and Bauer, U.M. (2009). PRMT1-mediated arginine methylation of PIAS1 regulates STAT1 signaling. Genes Dev 23, 118-132.

Webster, G.J., Reignat, S., Maini, M.K., Whalley, S.A., Ogg, G.S., King, A., Brown, D., Amlot, P.L., Williams, R., Vergani, D., et al. (2000). Incubation phase of acute hepatitis $B$ in man: dynamic of cellular immune mechanisms. Hepatology 32, 1117-1124.

Wei, C., Ni, C., Song, T., Liu, Y., Yang, X., Zheng, Z., Jia, Y., Yuan, Y., Guan, K., Xu, Y., et al. (2010). The hepatitis B virus $X$ protein disrupts innate immunity by downregulating mitochondrial antiviral signaling protein. J Immunol 185, 1158-1168.

Whitten, T.M., Quets, A.T., and Schloemer, R.H. (1991). Identification of the hepatitis $B$ virus factor that inhibits expression of the beta interferon gene. J Virol 65, 4699-4704.

Wieland, S., Thimme, R., Purcell, R.H., and Chisari, F.V. (2004). Genomic analysis of the host response to hepatitis B virus infection. Proc Natl Acad Sci U S A 101, 6669-6674.

Wieland, S.F., and Chisari, F.V. (2005). Stealth and cunning: hepatitis B and hepatitis C viruses. J Virol 79, 9369-9380.

Wu, J., Meng, Z., Jiang, M., Pei, R., Trippler, M., Broering, R., Bucchi, A., Sowa, J.P., Dittmer, U., Yang, D., et al. (2009). Hepatitis B virus suppresses toll-like receptor-mediated innate immune responses in murine parenchymal and nonparenchymal liver cells. Hepatology 49, 1132-1140.

Wu, M., Xu, Y., Lin, S., Zhang, X., Xiang, L., and Yuan, Z. (2007). Hepatitis B virus polymerase inhibits the interferon-inducible MyD88 promoter by blocking nuclear translocation of Stat1. J Gen Virol 88, 3260-3269.

Zeng, W., Sun, L., Jiang, X., Chen, X., Hou, F., Adhikari, A., Xu, M., and Chen, Z.J. (2010). Reconstitution of the RIG-I pathway reveals a signaling role of unanchored polyubiquitin chains in innate immunity. Cell 141, 315-330.

Zeng, W., Xu, M., Liu, S., Sun, L., and Chen, Z.J. (2009). Key role of Ubc5 and lysine-63 polyubiquitination in viral activation of IRF3. Mol Cell 36, 315-325.

Zhao, T., Yang, L., Sun, Q., Arguello, M., Ballard, D.W., Hiscott, J., and Lin, R. (2007). The NEMO adaptor bridges the nuclear factorkappaB and interferon regulatory factor signaling pathways. Nat Immunol 8, 592-600.

Zheng, D., Chen, G., Guo, B., Cheng, G., and Tang, H. (2008). PLP2, a potent deubiquitinase from murine hepatitis virus, strongly inhibits cellular type I interferon production. Cell Res 18, 1105-1113. 